Published in ACS Appl Mater Interfaces, 2015, 7, 21828-21834,

which should be cited when referring to this work

\title{
Visualization of Polymer Deformation Using Microcapsules Filled with
}

\section{Charge-Transfer Complex Precursors}

\author{
Anna Lavrenova, Jacob Farkas, Christoph Weder* and Yoan C. Simon*
}

Adolphe Merkle Institute, University of Fribourg, Chemin des Verdiers 4, 1700 Fribourg, Switzerland

\begin{abstract}
The visualization of polymer deformation using microcapsules filled with charge-transfer complex precursors is reported. Poly(urea-formaldehyde) microcapsules filled with toluene and either hexamethylbenezene as donor or chloranil as acceptor were prepared by an in-situ polymerization of an oil-in-water emulsion. The resulting microcapsules were incorporated in a poly(dimethylsiloxane) matrix by dispersing them in the monomer and subsequent curing. The microcapsules were shown to rupture when the polymer was placed under mechanical forces (i.e., tensile and compression), such that the donor and the acceptor are released into the polymeric matrix, where their combination to a charge-transfer complex results in a pronounced yellow-to-red color change. The factors influencing the extent of the color change (composition and mechanical strain) were investigated and a deformation threshold seems to be present. The new damage-sensing materials may be useful for a range of applications and the general concept applied seems to be readily applicable to other host polymers.
\end{abstract}

KEYWORDS: Charge-transfer complex, Damage sensing, Mechanochromic polymer, Microcapsule release system. Mechanically induced color change.

\section{INTRODUCTION}


Published in ACS Appl Mater Interfaces, 2015, 7, 21828-21834,

which should be cited when referring to this work

Mechanochromic polymers are macromolecular materials that change their absorption or/and fluorescence colors in response to mechanical deformation. ${ }^{1}$ Thanks to their ability to translate mechanical strain into a color change, these have emerged as an attractive class of stimuli-responsive materials and bear great promise for a variety of industrial applications, such as the detection of mechanical damage or pressure sensors. ${ }^{2,3}$ Several strategies have been utilized to impart mechanochromism to materials, ${ }^{1-4}$ including the covalent or noncovalent incorporation of mechanically sensitive units or "mechanophores" into a polymer of interest. In this case, elastic or plastic deformation of a polymer sample can trigger a conformational change, ${ }^{5}$ a change in chromophore interactions, ${ }^{6-9}$ or the reversible or irreversible breaking of covalent bonds, ${ }^{10-14}$ which in turn can lead to a (fluorescence) color change of the mechanophore in question. As an alternative to such molecular mechanisms, several mechanochromic materials based on dynamic photonic crystals have been investigated. ${ }^{15-17}$ In this case, the deformation changes the geometry of the spatially periodic variation of the multicomponent material, which in turn alters the photonic band gap, i.e., the frequency regime in which incident light is reflected.

A third possibility is the use of microcapsules that are filled with a dye precursor, which is released and activated when the protective shell is damaged. This general approach is particularly interesting because it allows one to impart functionality to a polymer of interest without the need to chemically modify the polymeric material per se. ${ }^{18}$ Instead, simple mixing of the active microcapsules with the polymer allows for the creation of functional materials. While the architecture is somewhat different, the same concept has been commercially exploited in carbonless copy paper, which is coated with gelatin microcapsules containing colorless leuco dyes. ${ }^{19}$ When pressure is applied, the dye microcapsules rupture, whereby the dye is released and can react with a developer residing outside the capsules to form a highly colored dye that leaves a permanent mark on the paper. Several researchers have also 
Published in ACS Appl Mater Interfaces, 2015, 7, 21828-21834,

which should be cited when referring to this work

exploited microcapsule-based release system in the context of damage sensing and/or selfhealing. Di Credico et al. encapsulated a UV-sensitive spiropyran into UV-absorbing microcapsules and incorporated these into a polyethylene glycol methacrylate photopolymer. ${ }^{20}$ Upon straining the material, the microcapsules ruptured and release their cargo and exposure to UV light led to the photo-induced electrocyclic ring-opening of the spiropyran dye to yield the merocyanine form, which in turn resulted in a local color change from yellow to red. White, Sottos, Moore and coworkers pioneered the use of monomer-filled microcapsules to impart polymers with self-healing capabilities. ${ }^{21}$ In this context, their groups explored the encapsulation of 1,3,5,7-cyclooctatetraene, a monomer that can undergo polymerization in the presence of the Grubbs-Love catalyst, and the microcapsules were incorporated into poly(acrylic acid). ${ }^{22}$ The authors observed a pronounced color change of the polymeric film after deformation and rupturing the microcapsules, which was attributed to the production of highly colored conjugated poly(acetylene) by ring-opening metathesis polymerization.

The examples reported in the literature suggest that dye-precursor filled microcapsules may be very useful to impart mechanochromic behavior to a broad range of polymers, but it appears that so far, few simple, chemistry-free chromophore systems have been explored. We thus embarked on the exploration of charge-transfer complexes (CTCs) as potential new indicators for damage sensing. CTCs are associations that are formed between an electron donor (D) and an electron acceptor (A), in which an electronic charge is transferred from the donor to the acceptor. ${ }^{23,24}$ This results in an electrostatic attraction that provides a stabilization force for the CTC, as well as the formation of a highly colored species. The possibility to instantly create a distinct color that is very different from the colors of the constituting donor and acceptor without a chemical reaction, the ability to tune this color via the choice of the D/A pair, and the fact that many useful donor and acceptor molecules are colorless makes the 
Published in ACS Appl Mater Interfaces, 2015, 7, 21828-21834,

which should be cited when referring to this work

approach very attractive for the visualization of mechanical damage in polymers. While it was recently shown that donor and acceptor molecules can be easily incorporated into microcapsules and that the rupture of such microcapsules can result in the formation of electrically conducting CTCs that may be useful to restore electrical conductivity, ${ }^{25}$ microcapsules filled with a D/A pair were to our best knowledge not yet embedded into a polymer matrix with the intent of using them as damage sensors.

Thus, poly(urea-formaldehyde) (PUF) microcapsules ${ }^{26}$ filled with toluene and either hexamethylbenezene (HMB) as donor or chloranil (CA) as acceptor (Figure 1) were prepared by an in-situ polymerization of an oil-in-water emulsion. The resulting microcapsules were incorporated in a poly(dimethylsiloxane) matrix by dispersing them in the monomer and subsequent curing. The microcapsules were shown to rupture when the polymer was placed under mechanical forces, such that the toluene solutions of the donor and the acceptor are released into the polymeric matrix, where their combination to a charge-transfer complex results in a pronounced yellow-to-red color change. The factors influencing the extent of the color change (composition and mechanical strain) were investigated and a deformation threshold seems to be present. The new damage-sensing materials may be useful for a range of applications and the general concept applied seems to be readily applicable to other D/A pairs, capsule materials, and host polymers. 


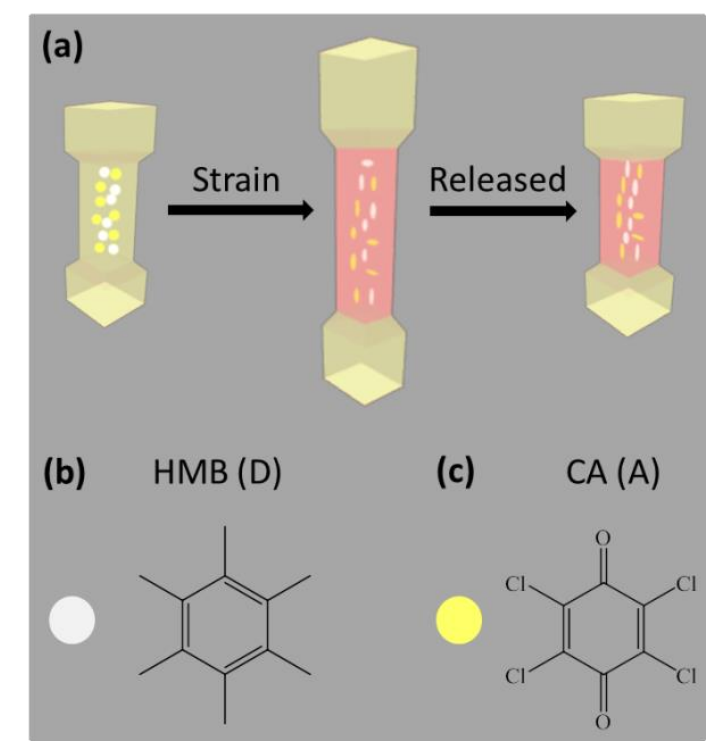

Figure 1. Schematic representation of the operating principle of a mechanoresponsive polymer (a) containing poly(urea-formaldehyde) (PUF) microcapsules filled with solutions containing donor (hexamethyl benzene, HMB) (b) and acceptor (chloranil, CA) (c) molecules. Straining the material results in the rupture of the microcapsules, leading to the release of donor and acceptor molecules and the formation of a colored charge-transfer complex (CTC).

\section{EXPERIMENTAL SECTION}

Materials. Urea, ammonium chloride, formaldehyde, resorcinol, chloranil (CA) and poly(ethylene-alt-maleic anhydride) $($ EMA $)\left(\mathrm{M}_{\mathrm{w}}=100,000-500,000 \mathrm{~g} / \mathrm{mol}\right)$ were all purchased from Sigma Aldrich and used without any purification. Hexamethylbenzene (HMB) and 1-octanol were purchased from ABCR and used as received. For the elastomeric matrix, poly(dimethylsiloxane) (PDMS) precursors were supplied by Dow Corning (Sylgard $\left.184^{\mathrm{TM}}\right)$. All solvents used in the preparation of the microcapsules were purchased from Sigma Aldrich and were of analytical grade.

Preparation of PUF Microcapsules Filled with Donor or Acceptor. The microcapsules used in this study were synthesized using a published in situ PUF microencapsulation 
Published in ACS Appl Mater Interfaces, 2015, 7, 21828-21834,

which should be cited when referring to this work

procedure to which small modifications were made. ${ }^{26}$ Prior to the encapsulation, EMA (0.67 g) was dissolved in deionized water $(75 \mathrm{~mL})$ at room temperature and stirred overnight. In parallel, HMB or CA $(500 \mathrm{mg})$ were dissolved in toluene $(60 \mathrm{~mL})$. The EMA solution was diluted with deionized water $(50 \mathrm{~mL})$ and placed in a $500 \mathrm{~mL}$ flask. The EMA solution was agitated at room temperature with a mechanical stirrer $(60 \mathrm{~mm}$ diameter) at $450 \mathrm{rpm}$ for the duration of the synthesis. Urea $(2.5 \mathrm{~g})$, resorcinol $(0.25 \mathrm{~g})$ and ammonium chloride $(0.25 \mathrm{~g})$ were then added to the flask. Once all compounds had dissolved, the $\mathrm{pH}$ was adjusted to 3.5 by drop-wise addition of a $1 \mathrm{M}$ aqueous $\mathrm{NaOH}$ solution and monitoring the $\mathrm{pH}$ with indicator paper. Subsequently, one drop of 1-octanol was added as a surface tension reducer and the HMB or CA solution $(60 \mathrm{~mL})$ was slowly poured into the reaction vessel. After stirring for 10 $\min , 6.3 \mathrm{~g}$ of formalin was added to the reaction mixture, the temperature was increased to 55 ${ }^{\circ} \mathrm{C}$, and the reaction mixture was stirred for another $4 \mathrm{~h}$ at $450 \mathrm{rpm}$. After this time, the agitation was stopped and the suspension was then cooled to room temperature and left to settle overnight. The resulting microcapsules were filtered off, rinsed with water, acetone and dichloromethane (DCM) and were air-dried for $24 \mathrm{~h}$.

Microcapsule Analysis. The average size of the microcapsules was determined by analyzing optical microscopy images (Figure 2). The dried microcapsules were dispersed in mineral oil, the dispersions were placed on glass microscopy slides, and images were acquired with an Olympus BX51 microscope mounted with a DP72 digital camera. The average diameter and standard deviation were determined from a data set of at least 200 of each type of microcapsules by using Stream Basic software. Only capsules that were clearly in focus were analyzed. Scanning electron microscopy (SEM) images of the surface of the dried microcapsules were obtained with a TE Scan Mira 3 after coating the samples with Pt/Pd (80/20) using a Cressington 208 HR high resolution sputter-coater. 
Published in ACS Appl Mater Interfaces, 2015, 7, 21828-21834,

which should be cited when referring to this work

Encapsulation Efficiency. A batch of microcapsules was prepared and the mass of the payload (HMB or CA) used in the reaction was recorded (Initial Payload Weight). After the synthesis was complete, the microcapsules were extensively washed with acetone and DCM in order to remove any payload that might have adsorbed on the capsule's surface. The microcapsules were then mechanically crushed with a mortar and pestle, washed with DCM and the suspension was filtered to separate the solid shell fragments from the dissolved payload. The filtrate was then dried under air overnight and the solid residue was weighed (Final Payload Weight) and compared to the amount fed into the reaction mixture, in order to determine the efficiency of the encapsulation process. No residual solvent could be observed by ${ }^{1} \mathrm{H}-\mathrm{NMR}$ and FTIR spectroscopies (vide infra). The encapsulation efficiency ((Final Payload Weight)/(Initial Payload Weight)*100\%) was 79\% and 67\% for HMB and CA, respectively.

Characterization of the Microcapsule Content. Fourier transform infrared spectroscopy (FTIR) spectroscopy was performed with a Perkin Elmer Spectrum 65 to characterize the payload incorporated into the microcapsules. ${ }^{1} \mathrm{H} \mathrm{NMR}$ data were recorded in $\mathrm{CDCl}_{3}$ at room temperature using a Bruker Avance III $360 \mathrm{MHz}$ NMR spectrometer. $\mathrm{HMB}$ and CA microcapsules were prepared and extensively washed with acetone and DCM to remove any payload that was not encapsulated. Afterwards the microcapsules were separately crushed and rinsed with DCM. The suspensions were filtered in order to eliminate the crushed microcapsule wall portion. The filtrates were collected, the solvents were removed in vacuo, and the remaining residues were analyzed by FTIR and 1H-NMR spectroscopy. The data were compared with the corresponding spectra of HMB or CA (Figure 3 and Supporting Figures S1 and S2). Further characterization was done by means of electron ionization mass spectrometry. Spectra of the extracted materials were compared with these of starting compounds (Supporting Figures S3-S6). 
Published in ACS Appl Mater Interfaces, 2015, 7, 21828-21834,

which should be cited when referring to this work

Preparation of Microcapsule-Containing Mechanochromic Polydimethylsiloxane. The samples were prepared by mixing the matrix material (Sylgard $184^{\mathrm{TM}}$ ) in the recommended ratio of 10 parts of the silicone base to 1 part of the curing agent with the desired total amount of the microcapsules (a total of $20 \% \mathrm{w} / \mathrm{w}$ ), pouring the mixture into a Teflon ${ }^{\circledR}$ Petri dish, and curing the resulting film by heating to $80{ }^{\circ} \mathrm{C}$ for $40 \mathrm{~min}$. While the size of samples varied depending on the experiments they were used for, a representative procedure is described here for specimens used in tensile experiments. The silicone base ( $15 \mathrm{~g})$ was mixed by hand with $3.3 \mathrm{~g}$ of microcapsules (1.65 $\mathrm{g}$ of PUF/HMB and $1.65 \mathrm{~g}$ of PUF/CA) in a Teflon ${ }^{\circledR}$ Petri dish (diameter $10 \mathrm{~cm}$ ). Then $1.5 \mathrm{~g}$ of the curing agent was added and the viscous mixture was stirred by hand and allowed to settle for ca. 5 minutes. Afterwards, the film was cured at 80 ${ }^{\circ} \mathrm{C}$ for $40 \mathrm{~min}$. Several compositions were prepared following this general procedure, including PDMS films containing the HMB and CA microcapsules in a ratio of 1:1, 1:2 and 2:1 and PDMS films containing only HMB or CA microcapsules that were used as the reference (total capsule content always $20 \% \mathrm{w} / \mathrm{w}$ ).

Mechanical Testing. All mechanical measurements were performed on Zwick/Roell Z010 tensile tester equipped with a $10 \mathrm{kN}$ load cell. The specimens were photographed before and after mechanical measurements. For tensile testing, dog-bone samples (cross-section: $80 \times 4.3$ $\times 4 \mathrm{~mm}$ ) were cut from premade films. In order to prevent the color change due to formation of charge-transfer complexes on the cut edges, the samples were allowed to stand for $24 \mathrm{~h}$ for the edge color to subside, presumably on account of photobleaching. Uniaxial tensile experiments were performed at a rate of $1 \mathrm{~mm} / \mathrm{min}$ by using DIN EN ISO 527-1 test standard. A biopsy puncher was used to prepare cylindrical samples with a diameter of $7.6 \mathrm{~mm}$ and height of $6 \mathrm{~mm}$ for the compression testing. In order to monitor color transitions at various compression ratios ( $0 \%$ to $80 \%)$, the samples were held at a desired compression ratio for 2 min, before they were released and photographed. 
Published in ACS Appl Mater Interfaces, 2015, 7, 21828-21834,

which should be cited when referring to this work

The formation of charge-transfer complexes was further confirmed by UV-Vis absorption spectroscopy using a Shimadzu UV-2401 PC. Spectra were recorded for the microcapsulecontaining PDMS films after applying tensile (30\%) or compressive (60\%) strains. Reference spectra were also recorded for an equimolar solution of $\operatorname{HMB}(0.021 \mathrm{M})$ and CA $(0.021 \mathrm{M})$ in toluene.

\section{RESULTS AND DISCUSSION}

Stimulated by literature investigations of this donor/acceptor pair in solution, ${ }^{27}$ and experiments that have shown CTC formation in blends with polymer matrices, ${ }^{28}$ we explored the suitability of a commercially available donor (hexamethylbenzene, HMB) and acceptor pair (chloranil, CA) shown in Figure 1. Initial solution experiments were conducted in order to investigate the change in optical properties upon CTC formation. Toluene solutions of each chromophore as well as an equimolar mixture (all $0.021 \mathrm{M}$ ) were prepared and their respective absorption spectra were recorded. HMB and CA both display absorption bands in the ultraviolet region (Supporting Figure S7) and are therefore colorless (HMB) or slightly yellow (CA). By contrast, the equimolar mixture of HMB/CA exhibited a deep-red color (Supporting Figure S7) on account of the appearance of a strong charge-transfer absorption band.

HMB and CA were thus separately encapsulated in poly(urea-formaldehyde) (PUF) core-shell microcapsules, using a well-established in situ emulsion polymerization method, ${ }^{26}$ which was slightly modified (the concentration of the wall-forming monomers was reduced by a factor of two) to reduce the agglomeration of the microcapsules by limiting the formation of a porous urea-formaldehyde layer on the capsule surface. ${ }^{29}$ Toluene was selected as the core solvent, because of its ability to efficiently solubilize both the donor and acceptor molecules. Furthermore its high boiling point allowed for high-temperature curing of the films. While the 
Published in ACS Appl Mater Interfaces, 2015, 7, 21828-21834,

which should be cited when referring to this work

targeted operating principle requires the encapsulation of only one of the D/A components (the second one could be simply dispersed or dissolved in the polymer matrix), the encapsulation of both components increases the fidelity of the process and more effectively prevents the formation of charge-transfer complexes prior to the application of mechanical stress. The choice of PUF as the encapsulation medium is rooted in the fact that PUF microcapsules are strong enough to remain intact during processing of the host polymer, but on the other hand, they can be easily broken when the matrix polymer is deformed. PUF microcapsules form quite effective barriers for liquids and therefore are widely utilized in academia and industry. ${ }^{30}$

Optical microscopy (Figure $2 \mathrm{a}$ and $2 \mathrm{~b}$ ) and scanning electron microscopy (Figure $2 \mathrm{c}$ and $2 \mathrm{~d}$ ) images reveal that the particles produced are spherical, closed, and that they have a relatively smooth surface. Evaluation of the microscopy images of 200 microcapsules revealed an average diameter of $157 \pm 26 \mu \mathrm{m}$ for the HMB/PUF microcapsules, while the diameter of CA/PUF microcapsules was $168 \pm 30 \mu \mathrm{m}$.
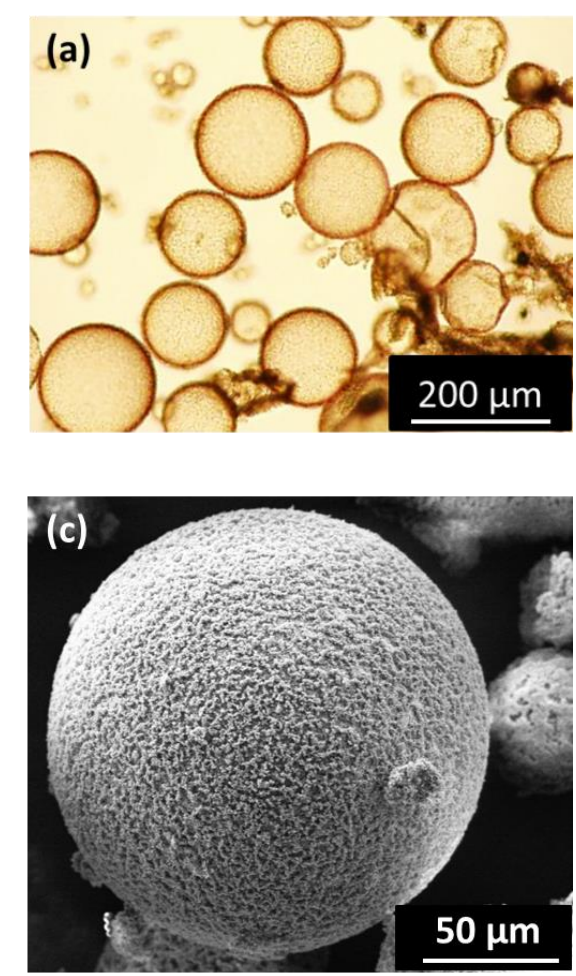
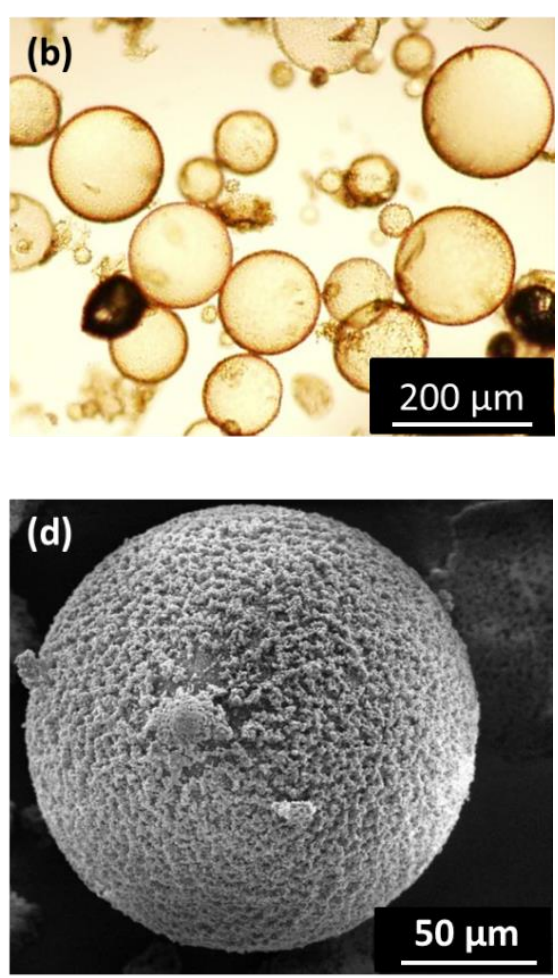
Figure 2. Optical micrographs of HMB/PUF (a) and CA/PUF (b) microcapsules. SEM images of single HMB/PUF (c) and CA/PUF microcapsules (d).

(a)

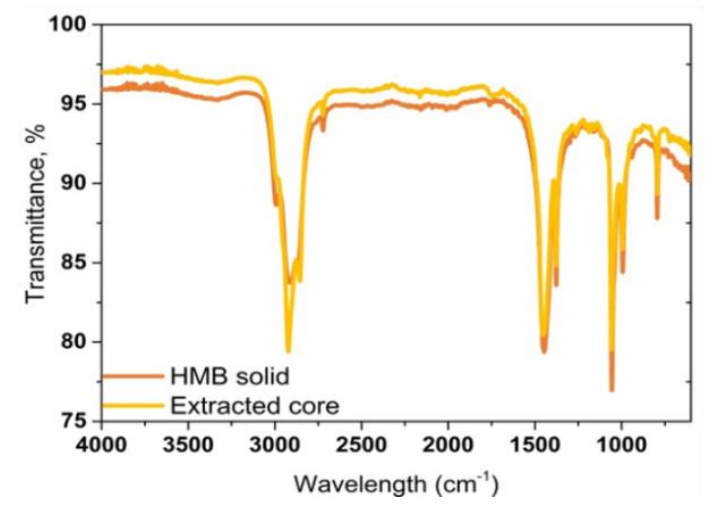

(b)

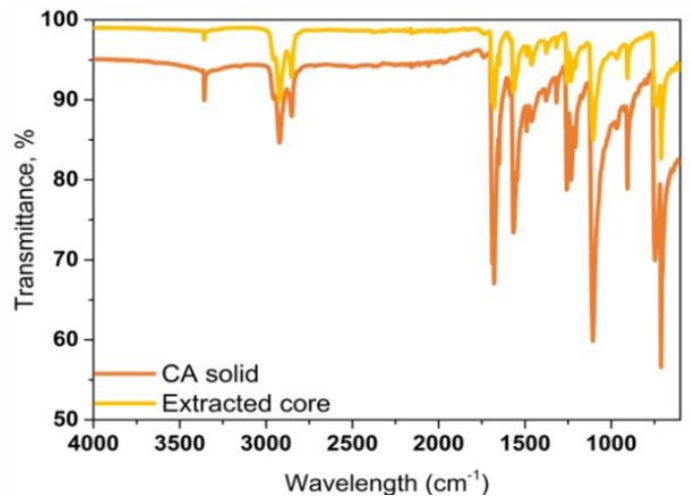

Figure 3. FTIR spectra of pristine HMB and HMB extracted from the HMB/PUF microcapsules (a). FTIR spectra of pristine CA and CA extracted from the CA/PUF microcapsules (b).

In order to confirm and quantify the encapsulation of the donor and acceptor molecules, respectively, into the microcapsules prepared, they were mechanically crushed and the payload was extracted with an organic solvent. Gratifyingly, FTIR spectra (Figure 3a and 3b), ${ }^{1} \mathrm{H}$ NMR spectra and mass spectra (Supporting Information, Figures S1-S6) confirm that the extracted payload consists exclusively of $\mathrm{HMB}$ and CA, respectively. Gravimetric analysis allowed a comparison of the amounts of donor and acceptor fed into the reaction mixtures and isolated from the microcapsules; this analysis resulted in an encapsulation efficiency of $79 \%$ and $67 \%$ for HMB and CA, respectively. Thus, these results clearly indicate a successful encapsulation of donor and acceptor molecules into PUF microcapsules.

In order to determine qualitatively if microcapsules filled with $\mathrm{HMB}$ and $\mathrm{CA}$ solutions, respectively, can serve as indicators of mechanical stress, the freshly prepared microcapsules were broken by shearing them between two glass slides. Figure 4 shows clearly that crushing HMB/PUF or CA/PUF microcapsules individually, does not impart any color change. 
However, when a mixture of $\mathrm{HMB} / \mathrm{PUF}$ and CA/PUF microcapsules was sheared, the characteristic red color of the HMB/CA CTC (Figure 4) was immediately apparent. ${ }^{31}$
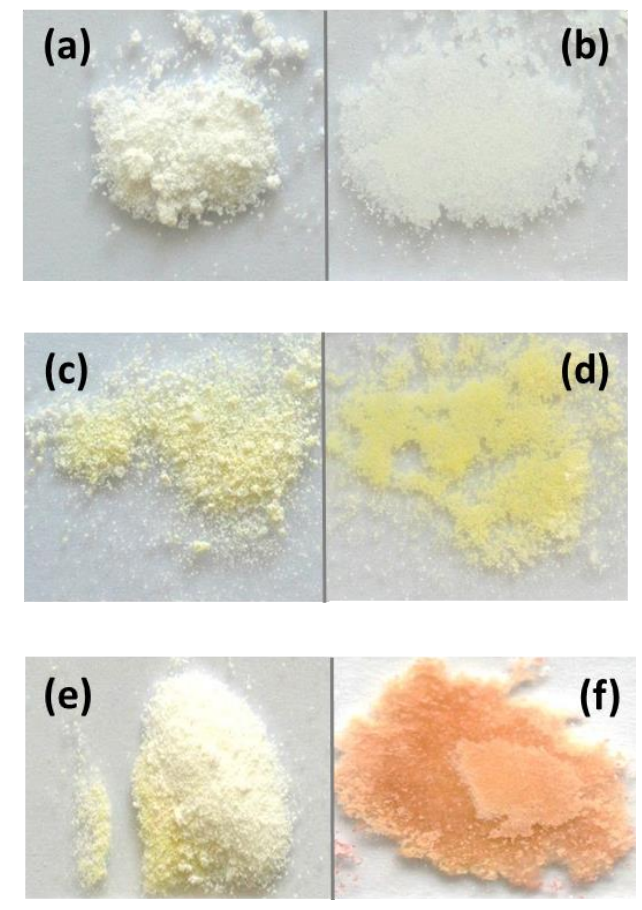

Figure 4. Photographs of HMB/PUF (a, b) and CA/PUF (c, d) microcapsules before (a, c) and after (b, d) breaking them by shearing between glass slides. Also shown is a mixture of HMB/PUF and CA/PUF microcapsules in their pristine (e) and broken (f) state.

In order to explore the possibility to utilize the CTC formation as strain or damage sensor in polymers, we elected to embed the HMB/PUF and CA/PUF microcapsule pair in a polydimethylsiloxane (PDMS) matrix. The choice of this matrix was motivated by its highly transparent nature, its high elasticity and reversible deformability, and the ability to readily embed the solvent-containing microcapsules in this matrix by mixing the prepolymer mixture with the microcapsules and subsequent curing at low temperature, which limits solvent evaporation. Also, the rupture of PUF microcapsules inside a PDMS matrix upon uniaxial tensile deformation was already described in the literature in the context of healing-agent filled capsules. ${ }^{32}$ While initial theoretical studies by White, Sottos and coworkers had shown crack deflection away from the capsules in linear elastic matrices using an Eshlby-Mura 
Published in ACS Appl Mater Interfaces, 2015, 7, 21828-21834,

which should be cited when referring to this work

equivalent inclusion model, ${ }^{21}$ they later demonstrated experimentally that a propagating tear could initiate capsule rupture in a nonlinear elastomeric matrix, like PDMS. In the present study, the overall microcapsule content was kept constant at $20 \% \mathrm{w} / \mathrm{w}$, the ratio of HMB/PUF to CA/PUF microcapsules was varied from $2: 1$ to $1: 1$ to $1: 2 \mathrm{w} / \mathrm{w}$. We note that even though PDMS is transparent, the capsule-containing films were quite hazy due to the scattering of the microcapsules (Figure 5).

In a first experiment, the response of the microcapsule-filled PDMS to a local stress such a cut was investigated. Thus, a PDMS film containing the HMB/PUF and CA/PUF microcapsules in a $1: 1 \mathrm{w} / \mathrm{w}$ ratio was cut with a razor blade, which was thought to result in microcapsule rupture and release of the D/A pair into the polymer matrix. Gratifyingly, within $1 \mathrm{~min}$, the damaged area displayed the characteristic red color of the HMB/CA CTC (Figure 5), whereas optical micrographs show the formation of red crystals upon rupture of the microcapsules (see Supporting Information, Figure S8). This color change is not only observed on the surface of the sample, but also along the entire cross-section that was damaged (Figure 5). As expected, no color change was observed when PDMS films containing either only HMB/PUF or CA/PUF microcapsules were damaged in a similar manner (Supporting Information, Figure S9).

(a)

(b)

(c)

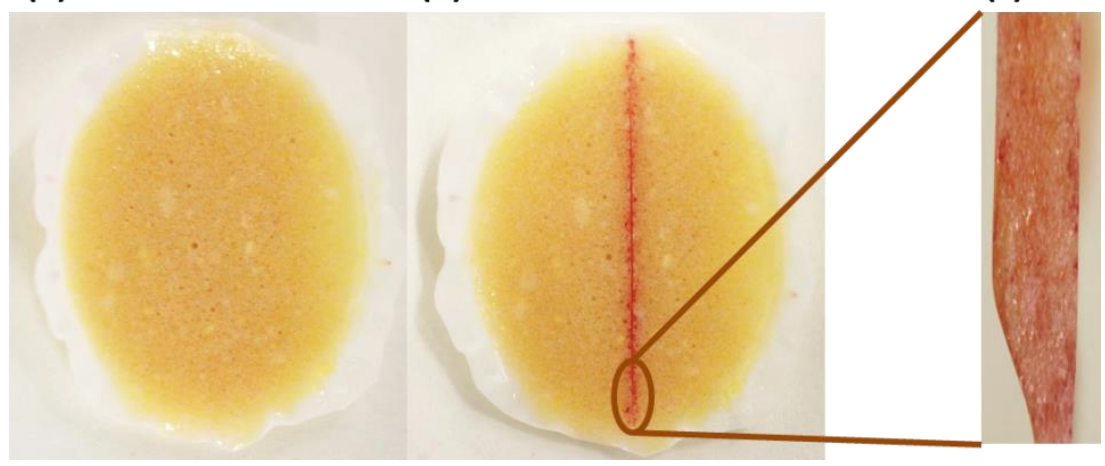


Published in ACS Appl Mater Interfaces, 2015, 7, 21828-21834,

which should be cited when referring to this work

Figure 5. PDMS film containing $10 \% \mathrm{w} / \mathrm{w}$ of each $\mathrm{HMB} / \mathrm{PUF}$ and CA/PUF microcapsules before (a) and after being cut with a razor blade (b). A magnification of the cross-section of the damaged area is shown as well (c).

We next explored the rupture of PUF microcapsules within the PDMS matrix under uniaxial deformation. Figures S10 represent the evolution of the tensile stress as a function of deformation for bare PDMS and capsule-loaded PDMS respectively and show an increase of tensile modulus in the presence of the capsules. In the context of a healable polymer system, it was shown previously that upon deformation beyond a stretched of $1.5 \%$, the walls of PUF microcapsules begin to fail and the payload is released. ${ }^{32}$ Thus, PDMS films containing $20 \%$ w/w of the HMB/PUF and CA/PUF microcapsules were stretched until failure (which usually occurred at an engineering strain of $>30 \%$ ) in order to ensure a maximum of payload release. While the overall microcapsule content was kept again constant at $20 \% \mathrm{w} / \mathrm{w}$, the ratio of HMB/PUF to CA/PUF microcapsules was varied from 2:1 to $1: 1$ to $1: 2 \mathrm{w} / \mathrm{w}$. Films containing more CA/PUF microcapsules were slightly more yellow in the pristine state due to the higher concentration of yellow CA. Therefore, even though for each of these compositions a significant color change from yellow to red was observed, when the films were damaged, the contrast was the highest for 2:1 $\mathrm{HMB} / \mathrm{PUF}$ to CA/PUF samples (Figure 6). Besides mere visual inspection, the assembly of HMB:CA CTCs was further corroborated by UV-Vis absorption spectroscopy (Figure 6). As discussed above, a dilute solution of equimolar amounts of HMB and CA in toluene displays a CTC band with maximum around ca. $520 \mathrm{~nm}$ (Figure 6). ${ }^{31}$ Prior to stretching, the latter was not observed in the UV-Vis spectra of any of the three compositions, whereas once the films were elongated this band appeared in the spectra, although the transition is a bit masked by the scattering caused by the PUF microcapsules. 

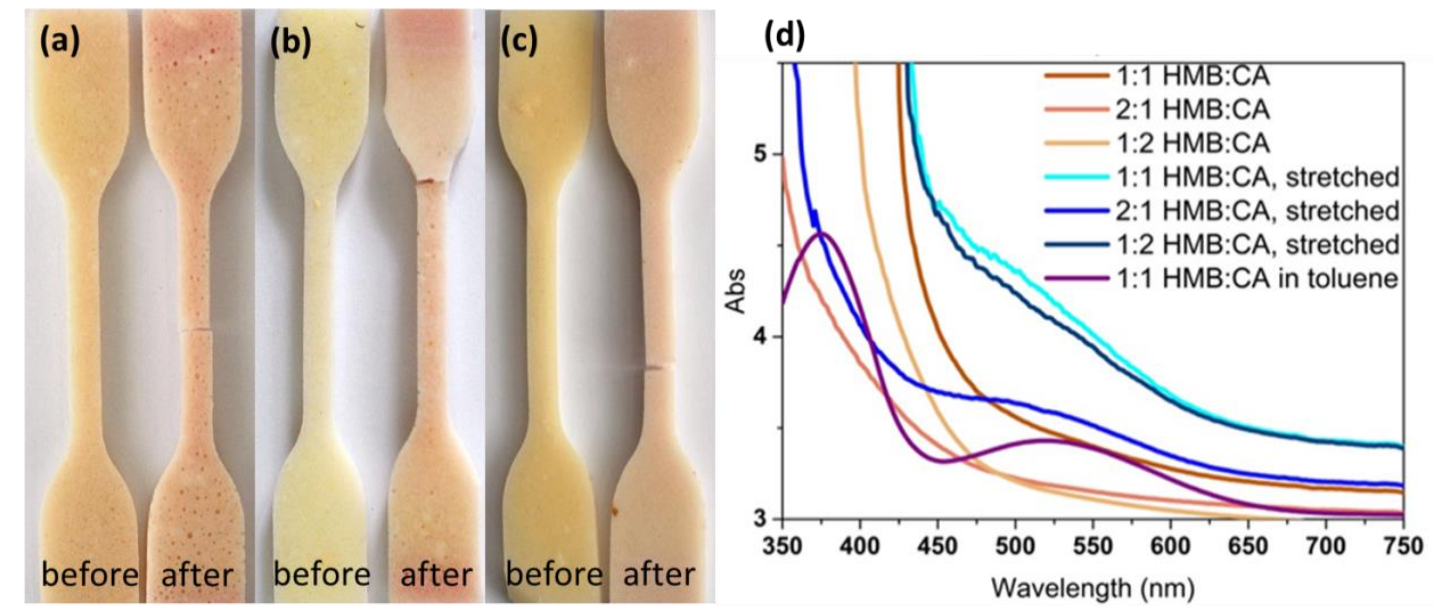

Figure 6. Photographs of PDMS films containing a total of $20 \% \mathrm{w} / \mathrm{w}$ of HMB/PUF and CA/PUF in a weight ratio of 1:1 (a), 2:1 (b) and 1:2 (c), before and after uniaxial deformation to break. UV-Vis absorption spectra (d) of these samples before and after deformation and of an equimolar solution of $\mathrm{HMB}$ and $\mathrm{CA}$ in toluene $(0.021 \mathrm{M})$ are also shown; the spectrum of the toluene solution was shifted vertically by an absorbance of 3 units.

Finally, the response of the microcapsule-filled PDMS samples to compressive forces was studied. Also in this case, the overall microcapsule content was kept constant at $20 \%$ w/w, and the ratio of HMB/PUF to CA/PUF microcapsules was varied as before. The cylindrically shaped samples were compressed to a desired compression ratio, kept compressed for 2 min in order to equilibrate the samples, before they were released and images were taken to record visual changes (Figure 7, Supporting Figure S11). It is to be noted that the capsule rupture is unlikely to be time dependent. Instead, the compression was only maintained here for 2 min to ensure that the desired compression ratio was attained and stable. The images show that compression to ratios of $<30 \%$ did not lead to any appreciable color change. This is consistent with the experiments of Keller and Sottos, who showed that an individual PUF capsule tends to fail at a displacement of more than $30 \% .{ }^{33}$ However, a clear color change towards red was observed when the films were compressed to ratios between $30 \%$ and $80 \%$; the samples failed at higher compression ratios. Note that even though the color change is 
Published in ACS Appl Mater Interfaces, 2015, 7, 21828-21834,

which should be cited when referring to this work

already observed at $30 \%$ compression ratio, it increased with increasing deformation up to $60 \%$, where saturation appears to have been reached. This could be attributed to the fact that at deformations below $60 \%$ not all of the microcapsules are broken; while at higher compression ratios a great majority of them must be broken and higher concentrations of the donor and acceptor are released into PDMS matrix. Similarly to the tensile experiments, samples containing a lower fraction of CA/PUF microcapsules displayed a better contrast between intact and damaged states, because the undeformed material is less yellow. In order to confirm the assembly of charge-transfer complexes under compression, samples deformed to $60 \%$ compression ratio (Supporting Figure S11) were investigated by UV-Vis absorption spectroscopy, which also in this case confirmed the formation of CTCs (Figure 7). It is interesting to note that regardless of the deformation speed, it took ca. $2 \mathrm{~min}$ for the color to appear, probably on account of the diffusion of the donor and acceptor molecules in the matrix. 

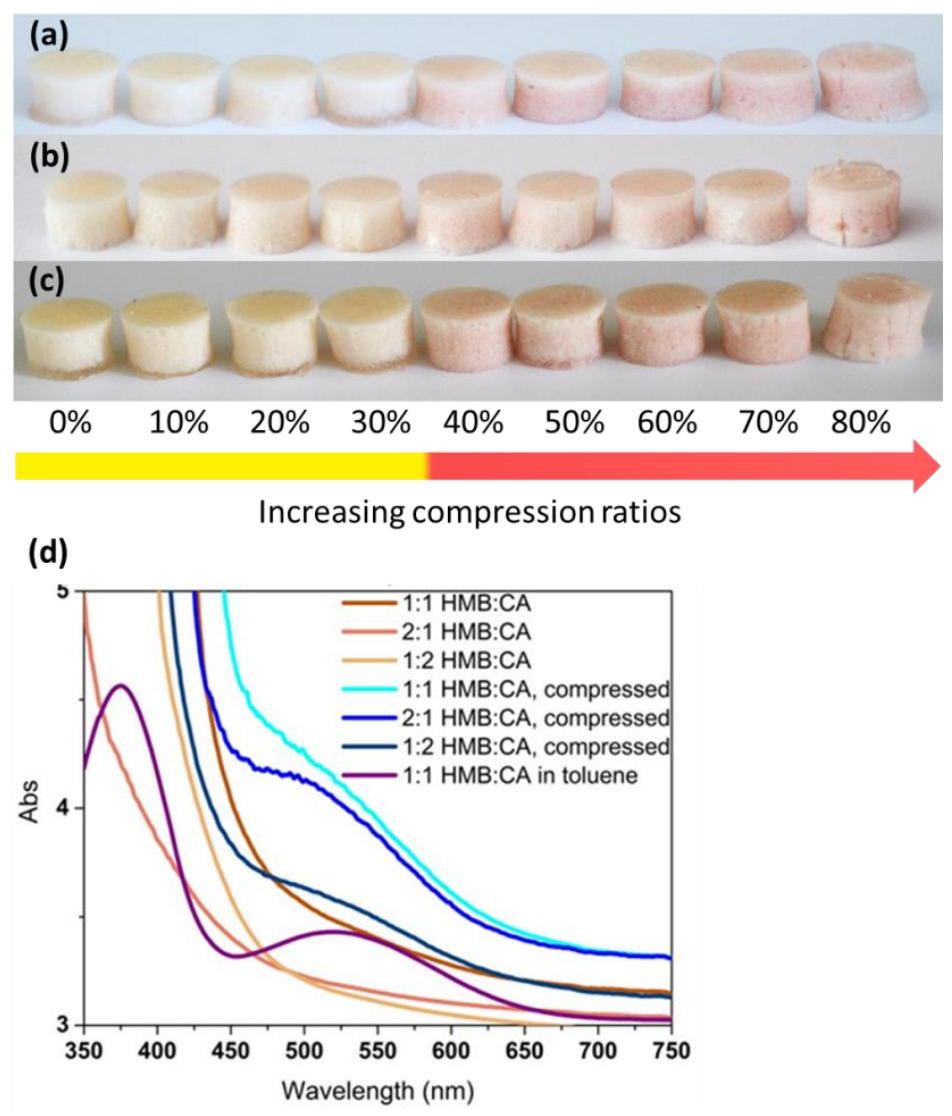

Figure 7. Images of PDMS films containing $20 \% \mathrm{w} / \mathrm{w}$ of HMB/PUF and CA/PUF in a weight ratio of $1: 1(\mathrm{a}), 2: 1$ (b) and 1:2 (c) after compression (2 min) to the compression ratio indicated in the figure. UV-Vis absorption spectra (d) of these samples before and after compression to $60 \%$ and of an equimolar solution of $\mathrm{HMB}$ and $\mathrm{CA}$ in toluene $(0.021 \mathrm{M})$ are also shown; the spectrum of the toluene solution was shifted vertically by an absorbance of 3 units.

\section{CONCLUSIONS}

In summary, a versatile method to create new mechanochromic strain-sensing materials was developed by encapsulating donor (hexamethylbenzene) and acceptor (chloranil) molecules into separate poly(urea-formaldehyde) (PUF) capsules and dispersing the latter into a rubbery polymer matrix. We demonstrated that the rupture of the capsules under tensile, compression or shear forces resulted in a pronounced color change of the polymeric films, easily detectable 
Published in ACS Appl Mater Interfaces, 2015, 7, 21828-21834,

which should be cited when referring to this work

by the naked eye. This color change was shown to be caused by the formation of CTCs of HMB and CA. Consequently, this strategy offers an attractive and scalable route that can, in principle, be readily implemented for a broad variety of donor/acceptor pairs and other solvents and matrices. To that end and in order to make this approach more widely applicable, current studies are aimed at increasing the shelf life of the capsules (i.e., through the use of less volatile solvents) and further extend this concept to other compounds (e.g., fluorescent and quencher moieties) and polymer matrices (e.g., epoxy resins).

\section{ASSOCIATED CONTENT}

\section{Supporting Information}

Additional ${ }^{1} \mathrm{H}$ NMR, mass and UV-Vis spectra together with tensile stress-strain curves, optical micrographs and photographs. This information is available free of charge via the Internet at http://pubs.acs.org/.

\section{AUTHOR INFORMATION}

\section{Corresponding Authors}

*Tel: +410263009465. Fax: +410263009624. E-mail: christoph.weder@ unifr.ch.

*Tel: +41263009311. Fax: +41263009624. E-mail: yoan.simon@unifr.ch.

\section{ACKNOWLEDGMENTS}

The authors are grateful to the European Research Council for the financial support (Grant ERC-2011-AdG 291490-MERESPO). This work was partially supported by the National Center of Competence in Research (NCCR) Bio-Inspired Materials, a research instrument of the Swiss National Science Foundation and the Swiss National Science Foundation. 
Published in ACS Appl Mater Interfaces, 2015, 7, 21828-21834,

which should be cited when referring to this work 


\section{REFERENCES}

1. Weder, C. Mechanochromic Polymers; In Encyclopedia of Polymeric Nanomaterials; Kobayashi, S.; Müllen, K., Eds.; Springer: Berlin Heidelberg, 2015; DOI 10.1007/978-3-64236199-9_6-4.

2. Weder, C. Polymers React to Stress. Nature 2009, 459, 45-46.

3. Caruso, M. M.; Davis, D. D.; Shen, Q.; Odom, S. A.; Sottos, N. R.; White, S. R.; Moore, J. S. Mechanically-Induced Chemical Changes in Polymeric Materials. Chem. Rev. 2009, 109, 5775-5798.

4. Haehnel, A. P.; Sagara, Y.; Simon, Y. C.; Weder, C. Mechanochemistry in Polymers with Supramolecular Mechanophores; In Top. Curr. Chem.; 2015; DOI 10.1007/128_2015_640.

5. Nallicheri, R. A.; Rubner, M. F. Investigations of the Mechanochromic Behavior of Poly(urethane-diacetylene) Segmented Copolymers. Macromolecules 1991, 24, 517-525.

6. Löwe, C.; Weder, C. Oligo(p-phenylene vinylene) Excimers as Molecular Probes:

Deformation-Induced Color Changes in Photoluminescent Polymer Blends. Adv. Mater. 2002, $14,1625-1629$.

7. Donati, F.; Pucci, A.; Cappelli, C.; Mennucci, B.; Ruggeri, G. Modulation of the Optical Response of Polyethylene Films Containing Luminescent Perylene Chromophores. $J$. Phys. Chem. B 2008, 112, 3668-3679.

8. Sagara, Y.; Komatsu, T.; Ueno, T.; Hanaoka, K.; Kato, T.; Nagano, T. Covalent Attachment of Mechanoresponsive Luminescent Micelles to Glasses and Polymers in Aqueous Conditions. J. Am. Chem. Soc. 2014, 136, 4273-4280.

9. Crenshaw, B. R.; Weder, C. Self-Assessing Photoluminescent Polyurethanes. Macromolecules 2006, 39, 9581-9589.

10. Davis, D. A.; Hamilton, A.; Yang, J.; Cremer, L. D.; Gough, D. V.; Potisek, S. L.; Ong, M. T.; Martinez, T. J.; White, S. R.; Moore, J. S.; Sottos, N. R. Force-induced Activation of Covalent Bonds in Mechanoresponsive Polymeric Materials. Nature 2009, 459, 68-72.

11. Craig, S. L. A Tour of Force. Nature 2012, 487, 176-177.

12. Gossweiler, G. R.; Hewage, G. B.; Soriano, G.; Wang, Q.; Welshofer, G. W.; Zhao, X.; Craig, S. L. Mechanochemical Activation of Covalent Bonds in Polymers with Full and Repeatable Macroscopic Shape Recovery. ACS Macro Lett. 2014, 3, 216-219.

13. Zhang, H.; Chen, Y.; Lin, Y.; Fang, X.; Xu, Y.; Ruan, Y.; Weng, W. Spiropyran as a Mechanochromic Probe in Dual Cross-Linked Elastomers. Macromolecules 2014, 47, 6783 6790 .

14. Peterson, G. I.; Larsenm, M. B.; Ganter, M. A.; Storti, D. W.; Boydston, A. J. 3DPrinted Mechanochromic Materials. ACS Appl. Mater. Interfaces 2015, 7, 577-583.

15. Foulger, S. J.; Jiang, P.; lattam, A.; Smith, D. W.; Ballato, J.; Dausch, D. E.; Grego, S.; Stoner, B. R. Photonic Crystal Composites with Reversible High-Frequency Stop Band Shifts. Adv. Mater. 2003, 15, 685-689.

16. Arsenault, A. C.; Clark, T. J.; Freymann, G. V.; Cademartiri, L.; Sapienza, R.; Bertolotti, J.; Vekris, E.; Wong, S.; Kitaev, V.; Manners, I.; Wang, R. Z.; John, S.; Wiersma, D.; Ozin, G. A. From Colour Fingerprinting to the Control of Photoluminescence in Elastic Photonic Crystals. Nat. Mater. 2006, 5, 179-184.

17. Kazmierczak, T.; Song, H.; Hiltner, A.; Baer, E. Polymeric One-Dimensional Photonic Crystals by Continuous Coextrusion. Marcomol. Rapid Commun. 2007, 28, 2210-2216. 
18. Esser-Kahn, A. P.; Odom, S. A.; Sottos, N. R.; White, S. R.; Moore, J. S. Triggered Release from Polymer Capsules. Macromolecules 2011, 44, 5539-5553.

19. White, M. A. The Chemistry Behind Carbonless Copy Paper. J. Chem. Educ. 1998, $75,1119-1120$.

20. Credico, B. D.; Griffini, G.; Levi, M.; Turri, S. Microencapsulation of a UV-

Responsive Photochromic Dye by Means of Novel UV-Screening Polyurea-Based Shells for Smart Coating Applications. ACS Appl. Mater. Interfaces 2013, 5, 6628-6634.

21. White, S. R.; Sottos, N. R.; Geubelle, P. H.; Moore, J. S.; Kessler, M. R.; Sriram, S.

R.; Brown, E. N.; Viswanathan, S. Autonomic Healing of Polymer Composites. Nature 2001, 409, 794-797.

22. Odom, S. A.; Jackson, A. C.; Prokup, A. M.; Chayanupatkul, S.; Sottos, N. R.; White, S. R.; Moore, J. S. Visual Indication of Mechanical Damage Using Core-Shell

Microcapsules. ACS Appl. Mater. Interfaces 2011, 3, 4547-4551.

23. Benesi, H. A.; Hildebrand, J. H. A Spectrophotometric Investigation of the Interaction of Iodine with Aromatic Hydrocarbons. J. Am. Chem. Soc. 1949, 71, 2703-2707.

24. Chen, E. C. M.; Wentworth, W. E. A Comparison of Experimental Determinations of Electron Affinities of pi Charge Transfer Complex Acceptors. J. Chem. Phys. 1975, 63, 31833191.

25. Odom, S. A.; Caruso, M. M.; Finke, A. D.; Prokup, A. M.; Ritchey, J. A.; Leonard, J. H.; White, S. R.; Sottos, N. R.; Moore, J. S. Restoration of Conductivity with TTF-TCNQ Charge-Transfer Salts. Adv. Funct. Mater. 2010, 20, 1721-1727.

26. Brown, E. N.; Kessler, M. R.; Sottos, N. R.; White, S. R. In Situ Poly(ureaformaldehyde) Microencapsulation of Dicyclopentadiene. J. Microencapsulation 2003, 20, 719-730.

27. Person, W. B. A Criterion for Reliability of Formation Constants of Weak Complexes. J. Am. Chem. Soc. 1965, 87, 167-170.

28. Kunzelman, J. N. Polymers with Integrated Sensing Capabilities. PhD Thesis Case Westn Rserve University, 2009.

29. Blaiszik, B. J.; Caruso, M. M.; McIlroay, D. A.; Moore, J. S.; White, S. R.; Sottos, N. R. Microcapsules Filled with Reactive Solutions for Self-healing Materials. Polymer 2009, 50, 990-997.

30. Andrade, B.; Song, Z.; Li, J.; Zimmerman, S. C.; Cheng, J.; Moore, J. S.; Harris, K.; Katz, J. S. New Frontiers for Encapsulation in the Chemical Industry. ACS Appl. Mater. Interfaces 2015, 7, 6359-6368.

31. Rathore, R.; Lindeman, S. V.; Kochi, J. K. Charge-Transfer Probes for Molecular Recognition Via Steric Hindrance in Donor-Acceptor Pairs. J. Am. Chem. Soc. 1997, 119, 9393-9404.

32. Keller, M. W.; White, S. R.; Sottos, N. R. A Self-Healing Poly(Dimethyl Siloxane) Elastomer. Adv. Funct. Mater. 2007, 17, 2399-2404.

33. Keller, M. W.; Sottos, N. R. Mechanical Properties of Microcapsules Used in a SelfHealing Polymer. Exp. Mech. 2006, 46, 725-733. 